\title{
VARIATIONAL BOUSSINESQ MODEL FOR SIMULATION OF COASTAL WAVES AND TSUNAMIS*
}

\author{
DIDIT ADYTIA \\ E. VAN GROESEN \\ Labmath-Indonesia, Lawangwangi, Bandung, Indonesia \\ \& Applied Mathematics,University of Twente, Enschede, Netherlands
}

\begin{abstract}
In this paper we describe the basic ideas of a so-called Variational Boussinesq Model which is based on the Hamiltonian structure of gravity surface waves. By using a rather simple approach to prescribe the profile of vertical fluid potential in the expression for the kinetic energy, we obtain a set of dynamic equations extended with one additional elliptic equation for the amplitude of the vertical profile. All expressions in the energy contain at most first order derivatives, which makes a numerical implementation with finite elements relatively easy. The applicability of the code is illustrated for two different applications in this paper. One application deals with tsunami simulations, for which we show the phenomenon of tsunami waveguiding before the coast of Lampung in Indonesia. Another application deals with simulations of coastal waves entering the small harbour of Cilacap on the south-coast of Java, Indonesia; we will show that the simulations indicate a resonance phenomenon in the small inner harbour.
\end{abstract}

\section{Introduction}

Coastal zones around the world are important because already from long times ago these are the places of large human settlements with major social and economic activities. Sustainable management of the coast includes tools to predict effects of natural or human interactions. In this paper, we consider two types of interactions: the relatively exceptional case of tsunamis generated by tectonic plate motions in the ocean before the coast, and the rather common case of investigating the effect of breakwaters to protect ships in a harbour against wind waves from sea or ocean. In these and other cases we have to rely on careful models and simulations to understand the ongoing processes in order to prevent or diminish the effects, or to investigate proposed changes or protection measurements. Such models and simulation tools become more and more common, but the phenomena are complicated and constant improvements and a careful use of the tools are needed. One of the problematic aspects of such phenomena from natural sciences is the presence of different scales in time and spaces, as is also the case for tsunamis and wind-wave simulations.

We will briefly describe the background of a Variational Boussinesq Model (VBM) and a finite element implementation for coastal waves in section 2. This VBM is based on the essential property that wave phenomena can be exactly described as a Hamiltonian system: the dynamics is completely determined by the total energy expressed in suitable

* This work is supported by a grant as part of the Scientific Collaboration IndonesiaNetherland, KNAW Mobility Project 07-MP-11. 
variables, just as in classical mechanics. As a consequences of the formulation, the numerical implementation guarantees conservation of the wave energy. In Section 3, we show results of tsunami simulations with additionally amplified wave height by the phenomenon of tsunami waveguiding. In Section 4, we deal with a harbour simulation and find a resonance phenomenon. We conclude in Section 5 with some remarks.

\section{Variational Boussinesq Model (VBM) and Finite Element (FE) implementation}

The equation of finite dimensional conservative dynamical systems, such as a harmonic oscillator, are found in Classical Mechanics as a Hamiltonian system which is derived from an action principle. Specifically, as critical points of the action functional given by

$$
\int\left[p \partial_{t} q-H(q, p)\right] d t
$$

where $H$ is the Hamiltonian, the total energy, which is the sum of potential and kinetic energy, expressed in position and momentum variables $q$ and $p$ respectively. For the harmonic oscillator with unit mass and frequency, the Hamiltonian is $H=\left(q^{2}+p^{2}\right) / 2$. In the same way, surface waves on the layer of inviscid, incompressible fluid under the influence of gravity can be obtained using the surface elevation $\eta$ and the surface fluid potential $\phi$ (functions of space and time) from the action principle for action functional:

$$
\int\left(\int \phi \partial_{t} \eta d x-H(\eta, \phi)\right) d t
$$

Here $x$ denotes the horizontal space variables, and $H$ is the total wave energy integrated over the spatial domain. This formulation follows from Luke's principle [1], and the corresponding equations can be written symbolically, using the variational derivative as the generalization of gradient of a function, as the Hamiltonian system

$$
\partial_{t} \eta=\delta_{\phi} H(\eta, \phi), \quad \partial_{t} \phi=-\delta_{\eta} H(\eta, \phi)
$$

This result shows that we can write the surface gravity waves solely in variables on the surface, i.e. the vertical depth coordinate $z$ does not appear, while all interior motions are completely and exactly accounted for. This reduction from a 3D spatial problem to a $2 \mathrm{D}$ problem is a great advantage for numerical simulations. Unfortunately, it is not possible to express $H$ explicitly in terms of $\eta$ and $\phi$. Nevertheless, the theoretical result above can still be used by finding approximations for $H$, and inserting the approximation in the action principle (2). The resulting approximate Hamiltonian system is then a consistent approximation, sharing the consequences of the exact formulation, such as exact energy conservation. Moreover, a further approximation by choosing a spatial approximation of the space dependent functions in the (approximate) Hamiltonian, leads to a consistent numerical discretization. 
The execution of the above program depends on the choice of the approximation of the Hamiltonian. Omitting details, see [2] for more information, the VBM was obtained by choosing approximations of the fluid potential in the interior as

$$
\Phi(x, z)=\phi(x)+F(z) \psi(x)
$$

with $F$ a function vanishing at the surface, $z=0$. For the choice $F=0$ we neglect all vertical variations, and eq.(3) become the well-known Shallow Water Equations (SWE), in which the dispersion is absent. By taking $F$ as a prescribed profile to introduce variation in vertical direction, for instance a parabolic profile or the potential profile of a linear harmonic wave with suitable wavelength, we introduce an approximate dispersion. The additional variable $\psi$ is a kind of amplitude variable in horizontal space variables. It is taken in an optimal way according to the action principle, namely by minimization of the Hamiltonian with respect to the additional variable $\psi$. It follows that $\psi$ has to satisfy

$$
\delta_{\psi} H(\phi, \eta, \psi)=0,
$$

which turns out to be a linear elliptic equation. The VBM is obtained by substituting the approximation (4) into the Hamiltonian. From the action principle in (2) we then obtain the Hamiltonian system (3) with the additional equation (5). The equation (5) is coupled with the time evolving $\eta$ and $\phi$, and has to be solved at each time step. Following this approach, it is found that the expression in the energy (Hamiltonian) contains at most first order derivatives. This makes it possible to use piecewise linear splines to obtain a relatively simple Finite Element (FE) implementation. By substituting such FE approximations into the Hamiltonian, we obtain a consistent FE discretization for the VBM. Disregarding small errors from numerical time marching, the spatial discretized energy is exactly conserved except for in- and out-flow effects. For the FE discretization we use an unstructured triangular grid. In the following we will illustrate the use of the linear version of the code.

\section{Tsunami waveguiding}

In this section we show results of a simulation above a realistic bathymetry for a synthetic tsunami source that leads to a tsunami that is running towards the south coast of Lampung where a very shallow area is present. Our main interest is to investigate if the phenomenon of waveguiding, introduced in ([3]) for synthetic bathymetry, can also be observed above a realistic bathymetry in this coastal area. The phenomenon itself is caused by the differences of wave speed above adjacent shallow and deep water areas in the propagation direction of the tsunami. This leads to large wave amplification, much larger than can be expected from possible differences in depth in the propagation direction.

In the coastal area South of Lampung, there is a very shallow, rather large, wedge-shaped area that is surrounded by deep water as can be seen in the left plot of Fig.1. For the numerical simulation, we used transparent boundary condition at the open ocean 
boundaries, and a hard-wall boundary condition at the coastline. The bathymetry data is taken from the one arc minute General Bathymetric Chart of Oceans (GEBCO).

To decide the location of the tsunami generation, we discussed with specialists about most likely area where a tsunami could be generated. This led to a position in the $4000 \mathrm{~m}$ deep area that is shown in the right plot of Fig.1. We consider an artificial initial wave (a bipolar hump with amplitude $2 \mathrm{~m}$ ) with a wavelength $\Lambda=40 \mathrm{~km}$ that is generated by a fast bottom excitation, so that the initial condition is released without speed.
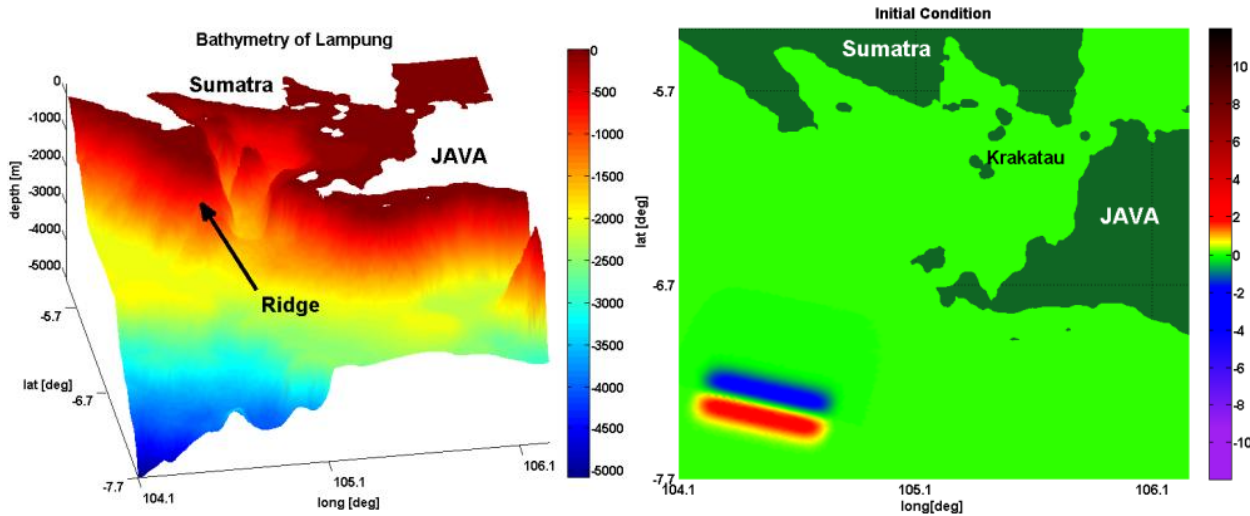

Figure 1. At the left, the bathymetry of Lampung is shown; notice the shallow area surrounded by deeper area at the south of Lampung. At the right, the shape and the location of the initial wave profile is shown.

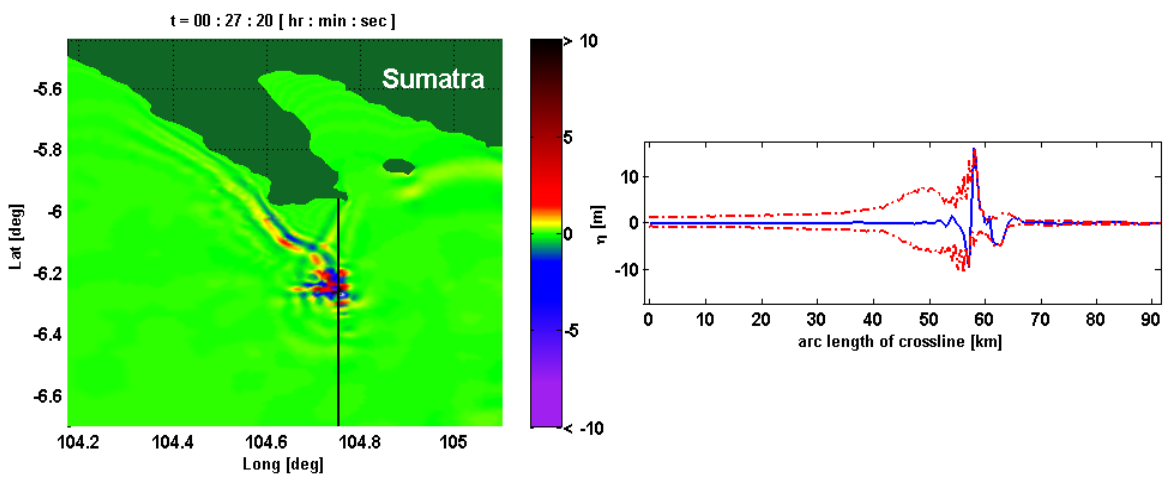

Figure 2. The surface elevation after 27 minutes of simulation is shown in the left plot. The elevation along the indicated cross section is shown at the right, with large wave heights when entering the shallow ridge area.

The snapshot of the tsunami elevation at the time when the tsunami reaches the shallow wedge, and the elevation along the cross section in Fig.2, show the appearance of extremely large surface elevations of more than $10 \mathrm{~m}$ high. This high wave can be followed in time and remains higher than the surrounding elevation, until at the coastline interaction with reflected waves leads to interference and very diverse wave heights. This result shows that the enhancement of wave entering the shallow region can be attributed to a waveguiding effect that is similar to observations in the synthetic case ([3]). 


\section{Harbour simulations}

Ocean waves effect the coast in many different ways. Here we will show some preliminary result of simulations near and in a harbour. We took as study case the harbour of Cilacap at the south-coast of Java, using a realistic bathymetry. For the FE implementation, the domain of simulation is discretized using an unstructured triangular grid as illustrated in left plot of Fig. 3; for the actual simulations we used a much finer grid with a gridsize of approximately $5 \mathrm{~m}$.
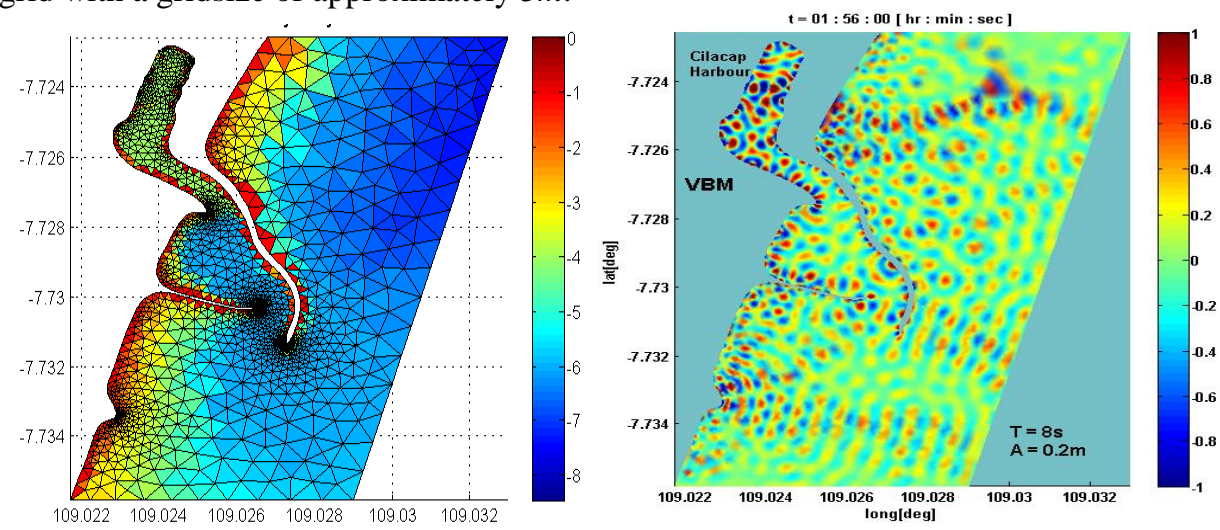

Figure 3. The left plot shows the unstructured triangular grid to approximate the bathymetry. The right plot shows a snapshot of the waves after almost two hours of simulation for the case of harmonic influx with $T=8 \mathrm{~s}$.

The harbour inside the breakwater consists of an inner harbour of approximately $4 \mathrm{~m}$ depth that is connected to an outer harbour of approximately $7 \mathrm{~m}$ depth; observe the narrow entrance to the inner harbour. We performed simulations for incoming harmonic waves from the south east by an influx perpendicular to the straight boundary at the right side in bathymetry plot (left plot of Fig. 3). This influx boundary is transparent for reflected waves. We consider influx for two periods, $T=8 \mathrm{~s}$ and $T=13 \mathrm{~s}$, corresponding to short and long waves of $60 \mathrm{~m}$ and $110 \mathrm{~m}$ respectively.

In this paper, we will mainly report about the result for shorter periods. The reason is, as we roughly calculated theoretically before the actual simulations were done, that near $T=8 s$ resonance takes place. For that period, the incoming wave profile generates a standing pattern in the total harbour area, but most dominantly in the small inner harbour. At the most extreme positions in the inner harbour, the waveheights are more than five times larger than the incoming wave. This is an overestimation of what can be expected realistically, because we choose to neglect bottom and wall friction. The incoming waves outside the harbour area interact with the reflected waves from the coast, and show a similar, but much less amplified, standing wave pattern in that part of the area. The period of the beating pattern is precisely the driving period of the incoming waves, see the time signal of the elevation in an extreme point in the inner harbour in Fig. 4. For $T=13 \mathrm{~s}$ the waves do not lead to a standing pattern. 

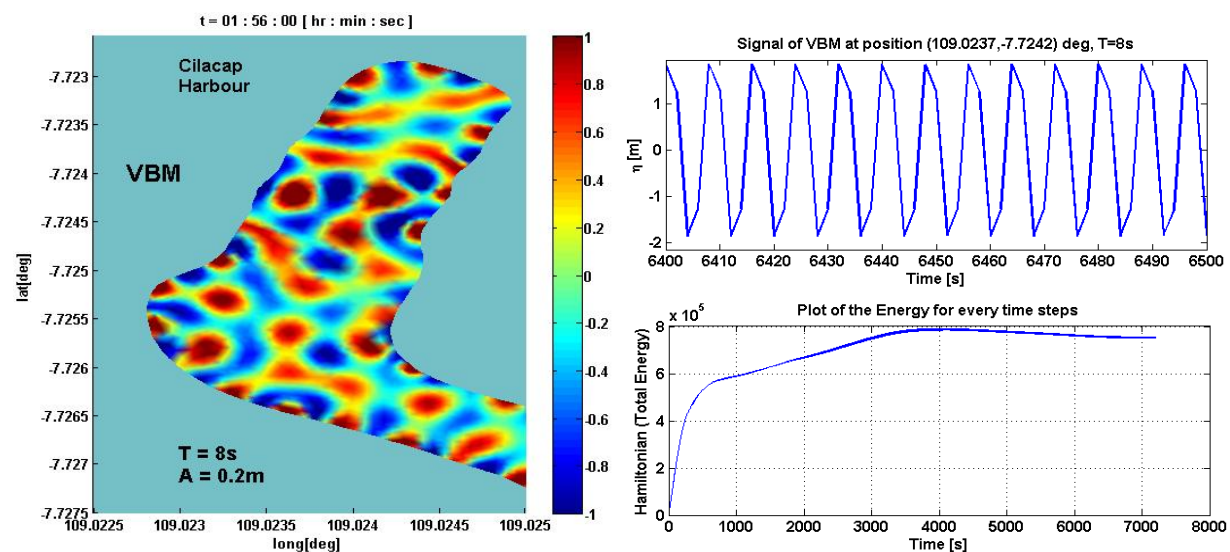

Figure 4. The snapshot of simulation in the inner harbour is shown in left plot. The upper right plot shows a signal of an artificial buoy data located in the inner harbour. The lower right plot shows the wave energy. Note that the energy is stable after $6500 s$.

Another interesting observation is about the numerical harbour loading. By this we mean the filling with waves of the initially still water in the harbour. In a numerical simulation, waves enter an undisturbed sea area, and it will take some transient period to get into a realistic situation, i.e. so that the effect of the artificial initially flat surface is not noticeable anymore. However, it is not easy to decide beforehand how much time this loading process takes. In our simulation, we calculated the wave energy over the total area of the numerical domain. Constancy of the total harbour energy serves as a measure of steadiness, since then the energy influx from the ocean is balanced by the energy outflow though the transparent boundaries. According to this measure, the loading time is long, and dependent on the influx period. For $T=13 \mathrm{~s}$, the energy reached a fixed level after 1 hour and 6 minutes (i.e. 307 wave periods), but for $T=8 s$, it took almost two hours (i.e. 900 wave periods), see Fig 4. This observation of long loading time has to be taken into account, to prevent that short simulation times give a wrong result.

\section{Conclusions and remarks}

In this paper we have described the basics of the Variational Boussinesq Finite Element Code, and have shown results of a tsunami simulation and of coastal waves penetrating into a small harbour. Both applications are characterized by large changes in wavelength. For the tsunami case this is because of the large difference in depth from the origin of excitation to the shallow coast, and for the harbour simulation because of the small inner harbour that has dimensions comparable to one wavelength of the ocean waves. Comparison with coarser grid sizes than used for this paper has shown that finer grids reveal aspects that are not, or much less pronounced, found on the coarser grids.

Another reason to investigate these cases was to see the effect of dispersion. Our Variational Boussinesq code has dispersion as explained in Section 2. We compared simulations of this code with simulations using the non-dispersive Shallow Water 
Equation (SWE). Both codes were implemented in a variationally consistent way, so both codes conserve exactly the total energy of that model, so that differences can only be attributed to the effect of dispersion.

For the tsunami simulation, Fig.6 shows that the maximal temporal amplitude at the coastline in the south of Lampung are quite similar for both codes, both with respect to maximal amplitudes as well as variability. For the harbour simulation, the situation is somewhat different. The resonance phenomenon that is shown here was not found for SWE code. This may be caused by the fact that resonance appears for different driving frequency, but all the simulations that we performed show different patterns between simulations with and without dispersion. In forthcoming publications we will present quantitative results of comparisons between results of our simulations with other codes and data measurements.

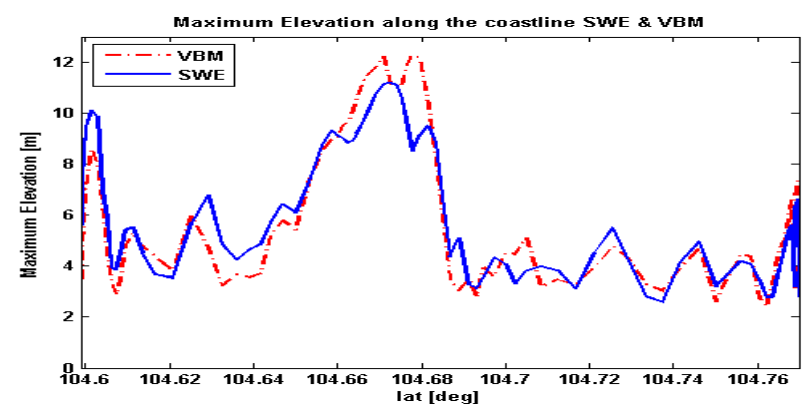

Figure 6. The maximum wave elevation along the coastline in the south of Lampung, for the Variational Boussinesq Code (dashed) and the SWE Code (solid).

\section{Acknowledgments}

This work is part of Mobility Programme 07-MP-11 in the Scientific Collaboration Indonesia-Netherlands funded by KNAW (Royal Netherlands Academy of Art and Sciences). We thank Prof. Sri Widiantoro, Dr. Hamzah Latief, Dr. Andonowati and Dr. Ardhasena Sopaheluwakan for discussions about different aspects of the problems.

\section{References}

1. J. C. Luke. J. Fluid Mech. 27, 395 (1967).

2. G. Klopman, M. Dingemans, and E. van Groesen. Proceeding of $22^{\text {th }}$ International Workshop on Water Waves and Floating Bodies, edited by Malenica and Senjanovi, I., Plitvice, Croatia. 125-128 (2007).

3. E. van Groesen, D. Adytia, and Andonowati. Natural Hazards and Earth System Sciences. 8, 175-185 (2008). 